\title{
Acute parietal lobe infarction presenting as Gerstmann's syndrome and cognitive decline mimicking senile dementia
}

This article was published in the following Dove Press journal:

Neuropsychiatric Disease and Treatment

2 July 2013

Number of times this article has been viewed

\author{
Tien-Yu Chen' \\ Chun-Yen Chen ${ }^{1,3}$ \\ Che-Hung Yen ${ }^{2,3}$ \\ Shin-Chang Kuo ${ }^{1,3}$ \\ Yi-Wei Yeh ${ }^{1,3}$ \\ Serena Chang' \\ San-Yuan Huang ${ }^{1,3}$ \\ 'Department of Psychiatry, \\ ${ }^{2}$ Department of Neurology, Tri-Service \\ General Hospital, School of Medicine, \\ National Defense Medical Center, \\ ${ }^{3}$ Graduate Institute of Medical \\ Sciences, National Defense Medical \\ Center, Taipei, Taiwan, Republic of \\ China
}

Correspondence: San-Yuan Huang

Department of Psychiatry, Tri-Service

General Hospital, School of Medicine,

National Defense Medical Center,

325 Cheng-Kung Road, Section 2,

Nei-Hu District, Taipei II4, Taiwan,

Republic of China

Tel + I I 886287927220

Fax +II 886287926715

Email hsy@ndmctsgh.edu.tw

\begin{abstract}
Gerstmann's syndrome encompasses the tetrad of finger agnosia, agraphia, acalculia, and right-left confusion. An elderly man with a history of several cardiovascular diseases was initially brought to the psychiatric outpatient department by his family because of worsening of recent memory, executive function, and mixed anxious-depressive mood. Gerstmann's syndrome without obvious motor function impairment and dementia-like features could be observed at first. Emergent brain computed tomography scan revealed new left-middle cerebral artery infarction over the left posterior parietal lobe. This case reminds us that acute cerebral infarction involving the parietal lobe may present as Gerstmann's syndrome accompanied by cognitive decline mimicking dementia. As a result, emergent organic workups should be arranged, especially for elderly patients at high risk for cerebral vascular accident.
\end{abstract}

Keywords: Gerstmann's syndrome, dementia, parietal lobe infarction

\section{Introduction}

Gerstmann's syndrome is a cluster of neurologic symptoms encompassing difficulty in writing, difficulty with arithmetic, difficulty in identifying fingers, and right-left confusion. It is associated with a lesion of the dominant angular gyrus region in the parietal lobe. ${ }^{1}$ Patients with Gerstmann's syndrome often present with other cognitive deficits, such as aphasia or any of a variety of apraxias. ${ }^{2}$ Some have also referred to this collection of symptoms as the angular gyrus syndrome. ${ }^{3}$ This syndrome may be associated with neoplasms of the central nervous system, ${ }^{4}$ neurodegenerative disease, and cerebrovascular disease. ${ }^{5}$

Here we report the case of an elderly patient who was brought to the psychiatric outpatient department by his family because of worsening of recent memory, executive function, and mixed anxious-depressive mood. The possibility of Gerstmann's syndrome was considered and the parietal lobe infarction was found later after examination. We discuss the management of this type of case to prevent misdiagnosis in the outpatient clinic.

\section{Case report}

$\mathrm{MrX}$, a 72-year-old right-handed male, had a history of heavy smoking, type 2 diabetes mellitus, hypertensive heart disease, complete atrioventricular block post implantation of a permanent pacemaker, and hyperlipidemia. He was under regular follow-up and taking a number of medications, including an amlodipine + benazepril combination, gliclazide, metformin, fluvastatin, and propranolol. The patient had shown signs of depression, anxiety, poor appetite, decreased interpersonal interaction, and poor quality 
of sleep in the previous 2 months. Although he could maintain a basic level of social function, his son mentioned that he had less interaction with family members and a mild recent memory deficit. One day, he was involved in a minor road traffic accident with no head injury, and later showed signs of worsening of short term memory, being unable to name family members, and of confabulation. The following day, his family brought him to the psychiatric outpatient department because of persistent dementia-like symptoms.

The family reported that the patient had agraphia, acalculia, and agnosia, with confabulation and a perplexed facial expression. He was still able to maintain attention, remained alert, and showed the normal range of psychomotor activity. No hallucination or obvious loss of muscle strength was found while we initially evaluated his condition. On account of his sudden decline in cognitive function, his advanced age, and multiple underlying medical conditions, our first consideration was cognitive function impairment of organic cause. Further workups, including routine blood biochemistry, cardiovascular investigation, and brain computerized tomography, were arranged immediately.

In addition to his worsening cognitive condition, the patient subsequently developed mild numbness and weakness of the right limbs. Neurologic examination revealed rightsided hemineglect of the visual field, a right-central type of facial palsy, mildly decreased muscle power over the right side $(4+/ 5+)$, increased deep tendon reflexes over the right knee and ankle region, a right-sided Babinski sign, and paresthesia over the right limbs.

The initial laboratory findings were as follows: white cell count $6,900 / \mu \mathrm{L}$; neutrophil to lymphocyte ratio 61:29; hemoglobin $14.9 \mathrm{~g} / \mathrm{dL}$; sodium $140 \mathrm{mmol} / \mathrm{L}$; potassium $3.6 \mathrm{mmol} / \mathrm{L}$; glutamic oxaloacetic transaminase $35 \mathrm{U} / \mathrm{L}$; glutamic pyruvic transaminase $44 \mathrm{U} / \mathrm{L}$; blood urea nitrogen $18 \mathrm{mg} / \mathrm{dL}$; creatinine $1.0 \mathrm{mg} / \mathrm{dL}$; total cholesterol $139 \mathrm{mg} / \mathrm{dL}$; and triglycerides $152 \mathrm{mg} / \mathrm{dL}$.

Electrocardiography showed sinus tachycardia and echocardiography showed hypokinesia of the left ventricle with an estimated ejection fraction of $40 \%-45 \%$. Duplex imaging of the neck arteries showed atherosclerotic changes located in the right common carotid artery $\mathrm{L} / 3$ to internal carotid artery with maximal stenosis in the internal carotid artery and percent diameter stenosis around $30 \%-50 \%$ and atherosclerotic changes in the left common carotid artery, $\mathrm{M} / 3$ to the internal carotid artery and external carotid artery with a percent diameter stenosis around $40 \%$. Brain computed tomography (Figure 1) showed acute ischemic infarcts over the left posterior parietal lobe in the left middle cerebral

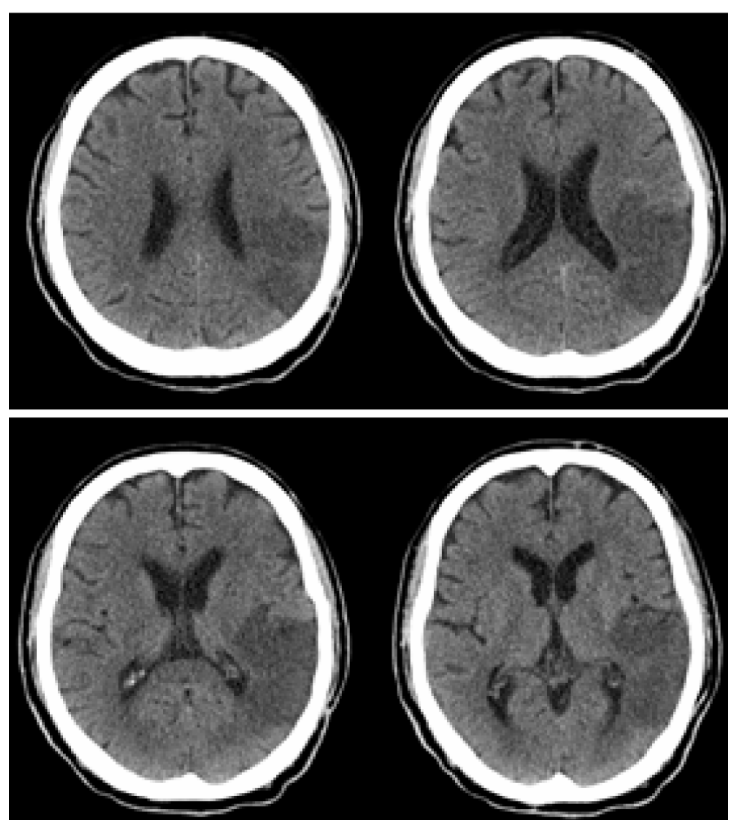

Figure I Brain computed tomography showed acute ischemic infarcts over the left posterior parietal lobe in the left middle cerebral artery territory without causing ventricular effacement or cerebral midline shift.

artery territory without causing ventricular effacement or cerebral midline shift. After emergent management in the emergency room, he was referred to the neurologic ward for further stroke management.

During hospitalization, the patient's speech was nonsensical but fluent. His construction of words and sentences was normal, fitting receptive aphasia. The patient also showed problems with reading, writing, calculations, and right-left disorientation, and was unable to recognize his fingers. All these symptoms indicated a diagnosis of Gerstmann's syndrome. The patient was unable to read short sentences, including his home address. Except for his name, the patient could not write down any meaningful sentences. However, his visuospatial performance as reflected by an ability to reproduce interlocking pentagons was relatively good. Further, he could not subtract 7 from 100 nor perform a serial 7 subtraction test. He also had problems with telling right from left when we asked him to touch his right knee with his left hand. He could not recognize his fingers when asked to raise one of them. The results of the Wechsler Adult Intelligence Quotient Scale-Revised showed mild mental retardation, with a full scale intelligence quotient (IQ) of 64, verbal IQ of 69, and a performance IQ of 59. Multilingual aphasia examinations showed that he was below the third percentile for naming and comprehension. His Mini-Mental Status Examination score was 9 points. On account of his Gerstmann's syndrome with receptive aphasia and alexia, occlusion at the parietal lobe 
was considered and this was consistent with brain computed tomographic imaging. ${ }^{6}$ Magnetic resonance imaging of the brain was not able to be performed because of implantation of a permanent pacemaker.

After eight days of hospitalization and treatment with clopidogrel, pentoxifylline, and rehabilitation therapy, he recovered most of his motor function. However, his receptive aphasia and impairment of cognitive function remained at the time of discharge.

\section{Discussion}

The left brain may be more dominant for calculations, logical ability, and hand preferences for right-handed individuals. The angular gyrus, located in the parietal lobe, influences the speech system. Damage to this area may give rise to alexia, aphasia, and Gerstmann's syndrome. ${ }^{7}$ Although we could not perform magnetic resonance imaging for better anatomic correlation due to his permanent pacemaker, computed tomography of the brain revealed a left middle cerebral artery infarction with left parietal encephalomalacia, but no obvious basal ganglia injury which explained why he could maintain his motor function. Because of his strokeinduced receptive aphasia, he had poor performance on IQ and language examinations.

When we first evaluated this elderly patient, all of his family members were concerned about his worsening cognitive function and signs of anxiety and depression. His stroke-induced Gerstmann's syndrome was easily mistaken as dementia or depression-related impairment of cognitive function. Organic problems were easily neglected in such cross-sectional evaluation and we should carefully clarify patient's past history and arrange further examinations if necessary. There has been a report of a patient with the angular gyrus syndrome that went unrecognized and was mistakenly treated as depressive pseudodementia. The reason for this was that the patient's cognitive function declined without impairment in motor function. ${ }^{8}$ Overlapping symptoms made it difficult for us to make a clear diagnosis in a short time. Several reports have suggested that neuroimaging studies should be performed immediately in the patients with atypical changes in mental status, for those over the age of 40 years with acute-onset impairment of cognitive function, ${ }^{9}$ confusion, or dementia of unknown cause, a first episode of psychosis of unknown etiology, a movement disorder of unknown etiology, anorexia nervosa, prolonged catatonia, and a first episode of major affective disorder or personality change after the age of 50 years. ${ }^{10}$ We arranged brain computed tomography for our patient because of his unusual symptoms and his high risk of cerebral infarction due to implantation of a permanent pacemaker for atrioventricular block, hyperlipidemia, and carotid artery stenosis.

Psychiatrists should also be aware that Gerstmann's syndrome is rarely seen in the psychiatric sector and may be a warning sign of parietal lobe infarction without any motor problems. The elderly with a high risk of cerebral vascular accident should be investigated carefully. We should not be overlooking any life-threatening conditions, especially in the outpatient department.

\section{Acknowledgments}

This study was supported by grants from National Science Council NSC101-2325-B16-003 (SYH); and by grants from Tri-Service General Hospital TSGH-C99-008-9- S01 (SYH), TSGH-C100-009-008-9- S01 (SYH); and by grants from Medical Affairs Bureau, Ministry of National Defense, Taiwan, DOD99-C04- 01 (SYH). DOD100-C09-01 (SYH). We thank Miss Yun-Hsin Lin for her assistance in the preparation of this manuscript.

\section{Disclosure}

The authors report no conflicts of interest in this work.

\section{References}

1. Gerstmann J. Some notes on the Gerstmann syndrome. Neurology. 1957;7(12):866-869.

2. Maeshima S, Okumura Y, Nakai K, et al. Gerstmann's syndrome associated with chronic subdural haematoma: a case report. Brain Inj. 1998;12(8):697-701.

3. Benson DF, Cummings JL. Angular gyrus syndrome simulating Alzheimer's disease. Arch Neurol. 1982;39(10):616-620.

4. Roux FE, Boetto S, Sacko O, et al. Writing, calculating, and finger recognition in the region of the angular gyrus: a cortical stimulation study of Gerstmann syndrome. J Neurosurg. 2003;99(4):716-727.

5. Zhou XQ, He R, Liu Z, et al. Clinical analysis of 7 patients with Gerstmann syndrome secondary to cerebral vascular disease. Zhongguo Yi Xue Ke Xue Yuan Xue Bao. 2002;24(5):510-511. Chinese.

6. Yang ZH, Zhao XQ, Wang CX, et al. Neuroanatomic correlation of the post-stroke aphasias studied with imaging. Neurol Res. 2008;30(4): 356-360.

7. Greenblatt SH. Alexia without agraphia or hemianopsia. Anatomical analysis of an autopsied case. Brain. 1973;96(2):307-316.

8. Nagaratnam N, Phan TA, Barnett C, et al. Angular gyrus syndrome mimicking depressive pseudodementia. J Psychiatry Neurosci. 2002;27(5):364-368.

9. Filley CM, Kleinschmidt-DeMasters BK. Neurobehavioral presentations of brain neoplasms. West J Med. 1995;163(1):19-25.

10. Weinberger DR. Brain disease and psychiatric illness: when should a psychiatrist order a CAT scan? Am J Psychiatry. 1984;141(12): 1521-1527. 


\section{Publish your work in this journal}

Neuropsychiatric Disease and Treatment is an international, peerreviewed journal of clinical therapeutics and pharmacology focusing on concise rapid reporting of clinical or pre-clinical studies on a range of neuropsychiatric and neurological disorders. This journal is indexed on PubMed Central, the 'PsycINFO' database and CAS.

The manuscript management system is completely online and includes a very quick and fair peer-review system, which is all easy to use. Visit http://www.dovepress.com/testimonials.php to read real quotes from published authors.

\footnotetext{
Submit your manuscript here: http://www.dovepress.com/neuropsychiatric-disease-and-treatment-journal
} 